\title{
Black Holes Thermodynamics in a new kind of Noncommutative Geometry
}

\author{
Mir Faizal $^{1}$, R. G. G. Amorim²,3, S. C. Ulhoa ${ }^{2,4}$ \\ ${ }^{1}$ Department of Physics and Astronomy, University of Waterloo, \\ Waterloo, Ontario, N2L 3G1, Canada. \\ ${ }^{2}$ Instituto de Física, Universidade de Brasília, 70910-900, \\ Brasília, DF, Brazil. \\ ${ }^{3}$ Faculdade Gama, Universidade de Brasília, \\ Setor Leste (Gama), 72444-240, \\ Brasília-DF, Brazil. \\ ${ }^{4}$ International Center of Condensed Matter Physics, \\ Universidade de Brasília, \\ 70910-900, Brasília, DF, Brazil.
}

\begin{abstract}
Motivated by the energy dependent metric in gravity's rainbow, we will propose a new kind of energy dependent noncommutative geometry. It will be demonstrated that like gravity's rainbow, this new noncommutative geometry is described by an energy dependent metric. We will analyse the effect of this noncommutative deformation on the Schwarzschild black holes and Kerr black holes. We will perform our analysis by relating the commutative and this new energy dependent noncommutative metrics using an energy dependent Moyal star product. We will also analyze the thermodynamics of these new noncommutative black hole solutions. We will explicitly derive expression for the corrected entropy and temperature for these black hole solutions. It will be demonstrated that for these deformed solutions black remnants cannot form. This is because these correction increase rather than reduce the temperature of the black holes.
\end{abstract}

\section{Introduction}

The renormalization group flow predicts that the constants in a quantum field theory will flow and depend on the scale at which such a theory is probed 11. However, the scale at which a theory is probed will in turn depend on the energy of the probe used in that theory. Hence, we can argue that the coupling constants depend on the energy of the probe. In fact, such scale dependence (energy dependence) of constants has been used for analyzing the flow of the cosmological constant [2] and the Newton constant [3]. It has thus been demonstrated using renormalization group flow that these constants depend on the scale at which a theory is probed, and so they depend on the energy of the 
probe. It may also be noted that string theory can be viewed as a two dimensional conformal field theory, and in this formalism the target space metric can be viewed as a matrix of coupling constants for this two dimensional conformal field theory. Hence, it is expected that this matrix of coupling constants will also flow, make the spacetime metric scale dependent. However, as the scale at which spacetime is probed will depend on the energy of the probe, it is expected that the spacetime metric will also become energy dependent. It may be noted that this energy dependent metric is the basis of gravity's rainbow [4, 5, 6, 77. As it is expected that spacetime geometry will be an emergent structure, it is not that surprising that the geometry will depend on the energy of the probe. Furthermore, in almost all theories of quantum gravity, for example, the discrete spacetime [8, models based on string field theory [9, spacetime foam [10, spin-network in loop quantum gravity (LQG) 11, noncommutative geometry [12, 13, and ghost condensation [14, the spacetime geometry is expected to break down at Planck scale, (hence at Planck energy). This can only occur if we have some dependence of scale in the metric, and this is achieved in gravity's rainbow by making the metric depend explicitly on the energy of the probe (and hence implicitly on the scale at which spacetime is probed).

Finally, we would like to comment that in gravity's rainbow, this energy (scale) dependence of the metric will only become effective at Planck energy, and in the IR limit this theory coincides with general relativity. Furthermore, this depends only on the energy of the probe, which fixes the scale at which the spacetime is probed, and not on the kind of particle used to probe the spacetime. It may be noted that constraints for such energy dependence of the metric from observational data has also been studied [15]. It has been argued that the energy of a particle emitted in Hawking radiation, near the horizon can be used to fix the scale at which the black hole is being probed, as this particle can act as an effective probe for the geometry 16. This can be used to incorporate the energy dependence in a black hole metric. It has also been demonstrated that this energy dependence of metric can alter the physics at the last stages of the evaporation of black holes [17, 18. This can also have important phenomenological consequences for the detection of mini black holes at particle collides [19. In the rainbow gravity, the energy dependence of the metric is usually incorporated through rainbow functions.

It may be noted that just like the Horava-Lifshitz gravity 20, 21, gravity's rainbow [4, 5, 6, 7] has also been proposed as a UV completion of general relativity. In the Horava-Lifshitz gravity [20, 21, the scaling properties of space and time are changed, and in the gravity's rainbow the metric of the spacetime becomes scale dependent. This scale dependence of the metric is incorporated by making the metric depend on the energy of the probe which probes the geometry of spacetime. In fact, it has been demonstrated that the gravity's rainbow and Horava-Lifshitz gravity are actually related to each other for specific choices of rainbow functions 22. The gravity's rainbow has also been studied using Finsler geometries $23,24,25,26,27$. Thus, there is a strong physical motivation to make the geometry dependent on the energy of the probe (and thus the scale at which the theory is probed). So, in this paper, we will propose an alternative way to incorporate this energy dependence in the geometry. We will propose that the parameter used to define the noncommutative deformation depends on the energy of the probe (hence the scale at which the spacetime is probed). In fact, as string theory can be used to motivate both the energy dependence of 
the emergent spacetime geometry and noncommutativity, it is possible to relate these two effects together. Furthermore, as the thermodynamics of rainbow black holes has been thoroughly studied [29, 30, 31, 32, 33, 34, 35, we will analyse the effect of this new proposal on the thermodynamics of black holes. In this structure, the noncommutativity will depend on the energy of the test particle. Hence, just like gravity acts differently on particles of different energies in gravity's rainbow, the deformation of gravity will be different for particles of different energies in this new theory of noncommutativity.

In this paper, we will analyse the effect of this new deformation on the thermodynamics of black holes. It may be noted that in the original proposal of black hole thermodynamics, the entropy scaled with the area of the horizon 36 , 37, 38, 39, 40. This observation has led to the development of the holographic principle [41, 42. In fact, the precise form of the area-entropy relation for black holes can be written as $S=A / 4$, where $S$ is the entropy of the black hole and $A$ is the area. However, this area-entropy relation is expected to get corrected as the black holes become small in size due to Hawking radiation. This is because the quantum fluctuations become important at that scale, and the quantum corrections in turn correct the black hole entropy. So, at small scales it is expected that the black hole thermodynamics will get modified, and this is also expected to modify the holographic principle [43, 44]. It may be noted that such corrections have been derived using various approaches, such as the non-perturbative quantum general relativity approach [45], the Cardy formula [46, 47, the partition function has been computed for BTZ black holes [48, and string theoretical effects [49, 50, 51, 52, 53. The noncommutativity also produces corrections terms in the black hole thermodynamic [54, 55, 56, 57]. The effect of noncommutativity is usually studied by replacing point-like structures in the original theory by objects which are smeared in spacetime. This is done by replacing the delta function by a Gaussian distribution with minimal width. The width of this Gaussian distribution is fixed by the noncommutative parameter. In this paper, we will analyse the black holes in this new noncommutative theory, which is motivated by gravity's rainbow. This will be done by following a procedure similar to the one used for analysing black hole thermodynamics in gravity's rainbow [16. Thus, we will first fix an energy scale from the energy of a particle near the horizon, and then use this energy scale to analyse the effects of this new noncommutativity on the thermodynamics of black holes.

\section{Energy Dependent Noncommutative Geome- try}

In this section, we will review the construction of noncommutative gravity [59, 60, 74, 75. In the noncommutative geometry, spacetime coordinates are promoted to a set of noncommutative self-adjoint operators, such that they satisfy

$$
\left[x^{\mu}, x^{\nu}\right]=i \theta^{\mu \nu},
$$

where $\theta^{\mu \nu}$ is a antisymmetric matrix. The product of two fields on this noncommutative spacetime can be replaced by a Moyal product of commutative fields, 
where the Moyal product is given by 76

$$
\begin{aligned}
f(x) * g(x) & =\left.\exp \left[\frac{i}{2} \frac{\partial}{\partial x^{\mu}} \theta^{\mu \nu} \frac{\partial}{\partial y^{\nu}}\right] f(x) g(y)\right|_{y \rightarrow x} \\
& =f(x) g(x)+\frac{i}{2} \theta^{\mu \nu} \partial_{\mu} f(x) \partial_{\nu} g(x)+\mathcal{O}\left(\theta^{2}\right) .
\end{aligned}
$$

It may be noted that the noncommutative field theories are nonlocal as the Moyal product of fields involves an infinite number of derivatives. Furthermore, as the spacetime coordinates do not commute, the noncommutativity gives rise to a minimum length in spacetime $\Delta x^{\mu} \Delta x^{\nu} \geq \frac{1}{2}\left|\theta^{\mu \nu}\right|$.

We can employ the Weyl quantization procedure [77, 78, to relate the metric tensor on noncommutative spacetime $\hat{g}_{\mu \nu}$ with the metric on commutative spacetime $g_{\mu \nu}$. Now the Fourier transform of the metric is given by

$$
\tilde{g}_{\mu \nu}(k)=\frac{1}{(2 \pi)^{2}} \int d^{4} x \exp \left(-i k_{\sigma} x^{\sigma}\right) g_{\mu \nu}(x),
$$

so, we can write

$$
\hat{g}_{\mu \nu}(\hat{x})=\frac{1}{(2 \pi)^{2}} \int d^{4} k \exp \left(i k_{\sigma} \hat{x}^{\sigma}\right) \tilde{g}_{\mu \nu}(k) .
$$

Thus, the operators $\hat{g}_{\mu \nu}$ and $\hat{x}$ replace the variables $g_{\mu \nu}$ and $x$. The product of two tensor fields on this noncommutative spacetime is equal to the Moyal product of two tensor fields on ordinary spacetime. So, the product of $\hat{f}_{\mu \nu}$ and $\hat{g}_{\mu \nu}$ is equal to $f(x)_{\lambda \sigma} * g(x)_{\tau \rho}$, such that [59, 60],

$$
\begin{aligned}
f(x)_{\lambda \sigma} * g(x)_{\tau \rho}= & \frac{1}{(2 \pi)^{4}} \int d^{4} k d^{4} p \exp \left[i\left(k_{\mu}+p_{\mu}\right) x^{\mu}-\frac{i}{2} k_{\mu} \theta^{\mu \nu} p_{\nu}\right] \\
& \times \tilde{f}_{\lambda \sigma}(k) \tilde{g}_{\tau \rho}(p) \\
= & \left.\exp \left[\frac{i}{2} \frac{\partial}{\partial x^{\mu}} \theta^{\mu \nu} \frac{\partial}{\partial y^{\nu}}\right] f_{\lambda \sigma}(x) g_{\tau \rho}(y)\right|_{y \rightarrow x}
\end{aligned}
$$

where $\tilde{f}_{\lambda \sigma}(k)$ is the Fourier transform

$$
\tilde{f}_{\lambda \sigma}(k)=\frac{1}{(2 \pi)^{2}} \int d^{4} x \exp \left(-i k_{\sigma} x^{\sigma}\right) f_{\lambda \sigma}(x) .
$$

In presence of matter the situation with regards to noncommutativity of spacetime coordinates can be imposed on the vierbeins as

$$
g_{\mu \nu}=e^{a}\left(\mu * e^{b}{ }_{\nu} \eta_{a b} .\right.
$$

Now motivated by gravity's rainbow, we will define a new energy dependent noncommutative geometry. We will also demonstrate that just like the gravity's rainbow, it will also produce an energy dependent metric. In gravity's rainbow, a one-parameter family of energy-dependent orthonormal frame fields give rise to a one-parameter family of energy-dependent metrics [4, 5, 6, 7]

$$
g^{\mu \nu}\left(E / E_{p}\right)=e_{a}^{\mu}\left(E / E_{p}\right) e_{b}^{\nu}\left(E / E_{p}\right) \eta^{a b},
$$


where $E$ is the energy of the test particle, and $E_{P}$ is the Planck energy. So, motivated by gravity's rainbow, let us assume that the commutativity depends on the energy of the test particle,

$$
\left[x^{\mu}, x^{\nu}\right]=i \theta^{\mu \nu}\left(E / E_{P}\right),
$$

where we require

$$
\lim _{E \rightarrow 0} \theta^{\mu \nu}\left(E / E_{P}\right) \rightarrow 0 .
$$

It may be noted that various different choices for $\theta^{\mu \nu}$ have been studied 80,81 . 82, 83. The noncommutativity is expected to arise because of background fluxes in string theory $[84,85$. It has also been demonstrated that certain background field can break the Lorentz symmetry of the system [86, 87. Furthermore, the Lorentz symmetry breaking has been studied for noncommutative field theories [88, 89, 90, 91. It is the breaking of Lorentz symmetry in the discrete spacetime [36], models based on string field theory [38, spacetime foam [39], spin-network in loop quantum gravity (LQG) 41, noncommutative geometry [12, 13, and ghost condensation 14, which has been used as a motivation to construct gravity's rainbow. Thus, motivated by this, we will now consider the case where the non-vanishing component of $\theta^{\mu \nu}\left(E / E_{P}\right)$ are $\theta^{23}\left(E / E_{P}\right)$ and $\theta^{32}\left(E / E_{P}\right)$. This would break the Lorentz symmetry in case of flat spacetime. We will now analyse the effect of such a noncommutative parameter on a Schwarzschild solution. So, we choose the following value for the noncommutative coordinate

$$
\theta^{\mu \nu}=\left[\begin{array}{cccc}
0 & 0 & 0 & 0 \\
0 & 0 & 0 & 0 \\
0 & 0 & 0 & \beta\left(E / E_{p}\right) \\
0 & 0 & -\beta\left(E / E_{P}\right) & 0
\end{array}\right],
$$

where we take the following simple energy dependent form for $\beta\left(E / E_{P}\right)$

$$
\beta\left(E / E_{P}\right)=\gamma\left(\frac{E}{E_{P}\left(E-E_{P}\right)}\right)^{\eta},
$$

where $\eta$ and $\gamma$ are parameters in the theory, which can be determined from experiments. It may be noted that

$$
\lim _{E \rightarrow 0} \beta\left(E / E_{P}\right) \rightarrow 0 .
$$

Thus, in the IR limit, we obtain the usual commutative theory, and only at very large energies, do we observe noncommutativity. Furthermore, the structure of spacetime breaks down at $E_{P}$, as

$$
\lim _{E \rightarrow E_{P}} \beta\left(E / E_{P}\right) \rightarrow \infty .
$$

Now $E_{P}$ is the maximum energy that any object can attain. The existence of a maximum energy scale has also been used as a motivation for the development of gravity's rainbow [4, 5, 6, 7]. The energy dependence is incorporated in gravity's rainbow using rainbow functions. Various rainbow functions have been proposed using different theoretical and experimental considerations [10, 15, 
92, 93. Using these rainbow functions different form of energy dependence of different geometry have been studied [16, 19, 17, 33. We have now observed that such a maximum energy scale can also be incorporated into this new kind of noncommutative geometry. However, we would like to point out that we have only used a very simple form of energy dependence, such that it statisfied the essential properties of such an energy dependent geometry. It is important to investigate other forms of energy dependent noncommutativity, and analyze its relation with other theoretical and experimental approaches. It would also be interesting to investigate if bounds for parameter $\eta$ and $\gamma$ can be obtained from different consideration similar to the bounds obtained for rainbow functions [15. However, in this paper, we will restrict our analysis to this simple form of energy dependence. We can also consider the simple case, when $\eta \sim \gamma \sim 1$, and so $\beta\left(E / E_{P}\right) \sim E / E_{P}\left(E-E_{P}\right)$.

Now we can define the Moyal product for such an energy dependent noncommutativity as

$$
\begin{aligned}
f(x)_{\lambda \sigma}\left[\otimes_{\left(E / E_{P}\right)}\right] g(x)_{\tau \rho}= & \exp \left[\frac{i}{2} \frac{\partial}{\partial x^{\mu}} \theta^{\mu \nu}\left(E / E_{P}\right) \frac{\partial}{\partial y^{\nu}}\right] \\
& \times\left. f_{\lambda \sigma}(x) g_{\tau \rho}(y)\right|_{y \rightarrow x}
\end{aligned}
$$

We can now also write the metric as

$$
g_{\mu \nu}=e_{(\mu}^{a}\left[\otimes_{\left(E / E_{P}\right)}\right] e^{b}{ }_{\nu} \eta_{a b} .
$$

It may be noted that just like gravity's rainbow, this metric is energy dependent.

The spin connection for this noncommutative spacetime can be written as

$$
\begin{aligned}
\omega_{\mu}^{a b}= & 2 e^{\nu a}\left[\otimes_{\left(E / E_{P}\right)}\right] \partial_{\mu} e_{\nu}^{b}-2 e^{\nu b}\left[\otimes_{\left(E / E_{P}\right)}\right] \partial_{\mu} e_{\nu}^{a} \\
& -2 e^{\nu a}\left[\otimes_{\left(E / E_{P}\right)}\right] \partial_{\nu} e_{\mu}^{b}+2 e^{\nu b}\left[\otimes_{\left(E / E_{P}\right)}\right] \partial_{\nu} e_{\mu}^{a} \\
& +e_{\mu c}\left[\otimes_{\left(E / E_{P}\right)}\right] e^{\nu a}\left[\otimes_{\left(E / E_{P}\right)}\right] e^{\sigma b}\left[\otimes_{\left(E / E_{P}\right)}\right] \partial_{\sigma} e_{\nu}^{c} \\
& -e_{\mu c}\left[\otimes_{\left(E / E_{P}\right)}\right] e^{\nu a}\left[\otimes_{\left(E / E_{P}\right)}\right] e^{\sigma b}\left[\otimes_{\left(E / E_{P}\right)}\right] \partial_{\nu} e_{\sigma}^{c}
\end{aligned}
$$

The spin connection is subject to the gauge transformation

$$
\left(\omega_{\mu}\right)_{b}^{a} \rightarrow\left[U_{C}\left[\otimes_{\left(E / E_{P}\right)}\right] \omega_{\mu}\left[\otimes_{\left(E / E_{P}\right)}\right] U_{C}^{-1}-\left(\partial_{\mu} U_{C}\right)\left[\otimes_{\left(E / E_{P}\right)}\right] U_{C}^{-1}\right]_{b}^{a},
$$

where $U_{C}$ is an element of a noncommutative group of orthogonal transformations $\operatorname{NCSO}(3,1)$ 60]. The noncommutative curvature tensor can be written as 59 .

$$
\begin{aligned}
\left(R_{\mu \nu}\right)_{b}^{a}= & \partial_{\mu}\left(\omega_{\nu}\right)_{b}^{a}-\partial_{\nu}\left(\omega_{\mu}\right)_{b}^{a}+\left(\omega_{\mu}\right)_{c}^{a}\left[\otimes_{\left(E / E_{P}\right)}\right]\left(\omega_{\nu}\right)_{b}^{c} \\
& -\left(\omega_{\nu}\right)_{c}^{a}\left[\otimes_{\left(E / E_{P}\right)}\right]\left(\omega_{\mu}\right)_{b}^{c}
\end{aligned}
$$

This noncommutative curvature tensor transforms as

$$
\left(R_{\mu \nu}\right)_{b}^{a} \rightarrow U_{C c}^{a}\left[\otimes_{\left(E / E_{P}\right)}\right]\left(R_{\mu \nu}\right)_{d}^{c}\left[\otimes_{\left(E / E_{P}\right)}\right]\left(U_{C}^{-1}\right)_{b}^{d} .
$$

The action for Einstein gravity deformed by noncommutativity is given by 59 , 60

$$
\begin{aligned}
S= & \frac{1}{2 \kappa} \int d^{4} x\left[e\left[\otimes_{\left(E / E_{P}\right)}\right] e_{a}^{\mu}\left[\otimes_{\left(E / E_{P}\right)}\right]\left(R_{\mu \nu}\right)_{b}^{a}\left[\otimes_{\left(E / E_{P}\right)}\right] e^{\nu b}\right] \\
& +\int d^{4} x\left[e\left[\otimes_{\left(E / E_{P}\right)}\right] \mathcal{L}_{\text {matter }}\right]
\end{aligned}
$$


where $e=\sqrt{-g}$. So, we can write the noncommutative Einstein equation as

$$
R_{\mu}^{a}-\frac{1}{2} R\left[\otimes_{\left(E / E_{P}\right)}\right] e_{\mu}^{a}=-\kappa T_{\mu}^{a},
$$

where

$$
\begin{aligned}
R_{\mu}^{b} & =\left(R_{\mu \nu}\right)^{a b}\left[\otimes_{\left(E / E_{P}\right)}\right] e_{a}^{\nu}, \\
R & =R_{\mu}^{a}\left[\otimes_{\left(E / E_{P}\right)}\right] e_{a}^{\mu},
\end{aligned}
$$

and $T_{\mu}^{a}$ is the energy momentum tensor for matter fields in this energy dependent noncommutative spacetime. It may be noted that this noncommutative metric induces a deformed diffeomorphism group. There exists a map between this deformed diffeomorphism group and the original diffeomorphism group [79. It is possible to obtain the solutions to the noncommutative Einstein equation by performing a noncommutative deformation of the solutions to usual Einstein equation [59, 60].

\section{New Noncommutativity for Black Holes}

In this section, we will analyse the noncommutative black hole solutions. This will be obtained by deforming the usual black hole solutions. The uncertainty principle, $\Delta p \geq 1 / \Delta x$, can be used to obtain a lower bound on the energy, $\Delta E \geq 1 / \Delta x$ of a particle in the Hawking radiation, which is near the horizon, and is used as a probe for the horizon. The value of the uncertainty in position can be taken to be the event horizon radius, $E \geq 1 / \Delta x \sim 1 / r_{+}$, where $r_{+}$is the radius of the horizon. This has been used to modify the thermodynamics of black holes in gravity's rainbow [16, 19, 17, 33. We shall use this energy to set the scale for the energy $E$ used in noncommutativity $\beta\left(E / E_{P}\right)$. Now we

will deform a Schwarzschild solution. We can write the original metric for a Schwarzschild solution as

$$
d s^{2}=-\left(1-\frac{2 M}{r}\right) d t^{2}+\left(1-\frac{2 M}{r}\right)^{-1} d r^{2}+r^{2}\left(d \theta^{2}+\sin ^{2} \theta d \phi^{2}\right),
$$

where $M$ is the mass of the black hole. Furthermore, we can write the tetrad field for this system, and deform it by the energy dependent noncommutativity given by Eq. (11), and obtain

$$
\sqrt{-\tilde{g}}=\sqrt{-g}+\beta^{2}\left(E / E_{p}\right) \sqrt{\frac{-g_{00} g_{11} g_{22}}{16 g_{33}}} \frac{\partial^{2} g_{33}}{\partial \theta^{2}} .
$$

Now we can calculate the entropy of the noncommutative Schwarzschild black hole and its temperature. The entropy of the noncommutative Schwarzschild black hole is given by

$$
\begin{aligned}
\tilde{S}\left(r_{+}\right) & =\left(1-\frac{\beta^{2}\left(E / E_{p}\right)}{4}\right) S\left(r_{+}\right) \\
& =\left(1-\frac{\gamma^{2}}{4}\left(\frac{E^{2}}{E_{P}^{2}\left(E-E_{P}\right)^{2}}\right)^{\eta}\right) S\left(r_{+}\right)
\end{aligned}
$$


where $S_{+}=A / 4$ is the entropy of the original commutative Schwarzschild black hole, and $\tilde{S}\left(r_{+}\right)$is the entropy of the noncommutative Schwarzschild black hole. The temperature is defined as $T^{-1}=\partial \tilde{S} / \partial M$, and so we can write the temperature of the noncommutative Schwarzschild black hole as

$$
\begin{aligned}
T & =\frac{1}{8 \pi M}\left(1+\frac{\beta^{2}\left(E / E_{p}\right)}{4}\right) \\
& =\frac{1}{8 \pi M}\left(1+\frac{\gamma^{2}}{4}\left(\frac{E^{2}}{E_{P}^{2}\left(E-E_{P}\right)^{2}}\right)^{\eta}\right) .
\end{aligned}
$$

As we can see the noncommutativity modifies the thermodynamics of the original Schwarzschild black hole. Here we have first obtained the corrections to the entropy of the Schwarzschild black hole, and then used these corrections to obtain the corrections to the temperature of the Schwarzschild black hole. It may be noted that for this deformed solution, a black remnant cannot form, as these correction increase rather than reduce the temperature of the black hole. We would also like to point out that black remnants form in gravity's rainbow only if an undeformed dispersion relation is used [17, 18, 19, and black hole remnants do not form if the modified dispersion relation are used 28. However, this argument cannot be used in this paper, as this paper only uses perturbative calculations.

Now we will study the thermodynamics of a noncommutative Kerr black hole. The general form of the line element of a spacetime with axial symmetry can be written as

$$
d s^{2}=g_{00} d t^{2}+2 g_{03} d \phi d t+g_{11} d r^{2}+g_{22} d \theta^{2}+g_{33} d \phi^{2},
$$

where the metric components are functions of $r$ and $\theta$. Now we can write the tetrad field for this system, and again the energy dependent noncommutativity given by Eq. (11). It will be useful to define, $\Delta g_{\mu \nu}=\tilde{g}_{\mu \nu}-g_{\mu \nu}$, and using this definition, we can write

$$
\tilde{S}=\frac{1}{4} \iint \sqrt{-g}\left[1+\frac{1}{2}\left(\frac{\Delta g_{11}}{g_{11}}+\frac{\Delta g_{22}}{g_{22}}\right)-\frac{g_{00} \Delta g_{33}}{2 \delta}\right] d \theta d \phi
$$

The metric for the Kerr black hole can be written as

$$
d s^{2}=-\frac{\psi^{2}}{\rho^{2}} d t^{2}-2 \frac{\chi \sin ^{2} \theta}{\rho^{2}} d t d \phi+\frac{\rho^{2}}{\Delta} d r^{2}+\rho^{2} d \theta^{2}+\frac{\Sigma^{2} \sin ^{2} \theta}{\rho^{2}} d \phi^{2}
$$

where $\Delta={ }^{2}+a^{2}-2 m r, \rho^{2}=r^{2}+a^{2} \cos ^{2} \theta, \Sigma^{2}=\left(r^{2}+a^{2}\right)^{2}-\Delta a^{2} \sin ^{2} \theta, \psi^{2}=$ $\Delta-a^{2} \sin ^{2} \theta, \chi=2 a m r$. Now we can use the standard procedure to calculate the corrections to the entropy of the Kerr black hole from noncommutativity. Thus, if $\tilde{S}\left(r_{+}\right)$is the entropy of the noncommutative Kerr black hole, then we can write,

$$
\begin{aligned}
\tilde{S}\left(r_{+}\right)= & S\left(r_{+}\right)-\frac{\pi \beta^{2}\left(E / E_{p}\right)}{8}\left\{8 a^{2}+\left[\frac{\left(a^{6}+2 a^{4} r_{+}^{2}-3 a^{2} r_{+}^{4}+2 r_{+}^{6}\right)}{a r_{+}\left(a^{2}+r_{+}^{2}\right)}\right]\right. \\
& \left.\times \tan ^{-1}\left(\frac{a}{r_{+}}\right)\right\} \\
= & S\left(r_{+}\right)-\frac{\pi \gamma^{2}}{8}\left(\frac{E^{2}}{E_{P}^{2}\left(E-E_{P}\right)^{2}}\right)^{\eta} \\
& \times\left\{8 a^{2}+\left[\frac{\left(a^{6}+2 a^{4} r_{+}^{2}-3 a^{2} r_{+}^{4}+2 r_{+}^{6}\right)}{a r_{+}\left(a^{2}+r_{+}^{2}\right)}\right] \tan ^{-1}\left(\frac{a}{r_{+}}\right)\right\},
\end{aligned}
$$


where $S\left(r_{+}\right)$is the entropy for the commutative black hole. Finally, we can write for the noncommutative Kerr black hole

$$
\begin{aligned}
\frac{\partial r_{+}}{\partial M}= & \frac{r_{+}}{r_{+}-M}, \\
\frac{\partial \tilde{S}}{\partial r_{+}}= & 2 \pi r_{+}+\frac{\pi \beta^{2}\left(E / E_{p}\right)}{8}\left\{\left[\frac{\left(a^{6}+2 a^{4} r_{+}^{2}-3 a^{2} r_{+}^{4}+2 r_{+}^{6}\right)}{r_{+}\left(a^{2}+r_{+}^{2}\right)^{2}}\right]\right. \\
& \left.+\left[\frac{\Phi}{a r_{+}^{2}\left(a^{2}+r_{+}^{2}\right)^{2}}\right] \tan ^{-1}\left(\frac{a}{r_{+}}\right)\right\},
\end{aligned}
$$

where $\Phi=a^{6}\left(a^{2}+3 r_{+}^{2}\right)-2 a^{4} r_{+}^{2}\left(a^{2}-r_{+}^{2}\right)+3 a^{2} r_{+}^{4}\left(3 a^{2}+r_{+}^{2}\right)-2 r_{+}^{6}\left(5 a^{2}+3 r_{+}^{2}\right)$. So the temperature for the noncommutative Kerr black hole can be written as

$$
\begin{aligned}
T^{-1}= & \left(\frac{\partial \tilde{S}}{\partial r_{+}}\right)\left(\frac{\partial r_{+}}{\partial M}\right), \\
= & {\left[\frac{r_{+}}{r_{+}-M}\right]\left[2 \pi r_{+}+\frac{\pi \beta^{2}\left(E / E_{p}\right)}{8}\left\{\left[\frac{\left(a^{6}+2 a^{4} r_{+}^{2}-3 a^{2} r_{+}^{4}+2 r_{+}^{6}\right)}{r_{+}\left(a^{2}+r_{+}^{2}\right)^{2}}\right]\right.\right.} \\
& \left.\left.+\left[\frac{\Phi}{a r_{+}^{2}\left(a^{2}+r_{+}^{2}\right)^{2}}\right] \tan ^{-1}\left(\frac{a}{r_{+}}\right)\right\}\right] . \\
= & {\left[\frac{r_{+}}{r_{+}-M}\right]\left[2 \pi r_{+}+\frac{\pi \gamma^{2}}{8}\left(\frac{E^{2}}{E_{P}^{2}\left(E-E_{P}\right)^{2}}\right)^{\eta}\right.} \\
& \times\left\{\left[\frac{\left(a^{6}+2 a^{4} r_{+}^{2}-3 a^{2} r_{+}^{4}+2 r_{+}^{6}\right)}{r_{+}\left(a^{2}+r_{+}^{2}\right)^{2}}\right]\right. \\
& \left.\left.+\left[\frac{\Phi}{a r_{+}^{2}\left(a^{2}+r_{+}^{2}\right)^{2}}\right] \tan ^{-1}\left(\frac{a}{r_{+}}\right)\right\}\right] .
\end{aligned}
$$

It may be noted that for this deformed solution, a a black remnant cannot form, as these correction also increase rather than reduce the temperature of the black hole.

Thus, we have obtained an expression for the corrections to the thermodynamics of Kerr black holes. Here the metric, and the thermodynamics of the black hole depends on the energy of the particle in Hawking radiation. It is known that the energy of the particle in the Hawking radiation is different for an in-falling observer than an asymptotic observer 94, 95, 96. So, the geometry of the spacetime will also appear considerable different for the two observes. It may be noted that the idea that the geometry of spacetime appear different for an in-falling observer and an asymptotic observer has been made for discussing the black hole complementarity $97,98,99,100$. It has been observed that the gravity's rainbow also modifies the geometry of a black hole, and this modification has been used to address the black hole information paradox [101, 102, 103. It may be noted in the commutative limit these corrections vanish, and we obtain the original case back. 


\section{Conclusion}

In this paper, motivated by gravity's rainbow, we have proposed a new kind of energy dependent noncommutative geometry. We have studied the noncommutative Schwarzschild black holes and the noncommutative Kerr black holes, using this new energy dependent noncommutative geometry. This was done by first using Weyl quantization procedure [77, 78, to relate the metric tensor on noncommutative spacetime with the metric on commutative spacetime. The relations thus derived were used specifically to obtain expressions for the corrections to the Schwarzschild metric and the Kerr black metric due to noncommutativity. Furthermore, we analyzed the thermodynamics of these noncommutative black holes. So, explicit expressions for the corrected entropy and temperature of these black hole solutions were obtained. These corrections vanished when the noncommutative parameter is set to zero, and hence, we derive the original commutative results back. It was observed that for these deformed solutions, black remnants cannot form. This is because these correction increase rather than reduce the temperature of the black holes.

It may be noted that this work take a different approach from the earlier works where the effect of noncommutativity has been studied by replacing pointlike structures in the original theory by objects which are smeared in spacetime [54, 55, 56, 57. The noncommutativity mixes ultraviolet and infrared divergences 58. It also incorporates non-locality in a controllable way 59. It has been studied in the context of string theory. This is because it is known that the transverse coordinates of D-branes can be regarded as matrices, and these matrices do not commute 61. Noncommutativity also occurs in the context of M-theory and 62,63 . In this analysis the compactification on the noncommutative torus has been studied. It has been demonstrated that deforming the commutative torus to the noncommutative torus corresponds to have a constant background three form potential. In fact, there are two commutative tori associated with a noncommutative torus, one to its odd and one to its even cohomology, leading to two commuting actions on the Teichmuller space. Noncommutative geometry has also been studied in the context of open strings ending on the D-branes. In this context gauge theories on noncommutative tori will appear as D-brane world volume theories [64. The D0-branes in type IIA string theory with a background two form field have also been studied, and it has been observed that the background two form field modifies the replacing ordinary multiplication by a noncommutative product 65. It has been demonstrated that there is a link between a noncommutative gauge theory and an ordinary gauge theory [71]. In fact, a relation between the noncommutative instantons and the ordinary instantons for Yang-Mills theory has also been observed 66. The relation between instantons on branes and the noncommutative Yang-Mills theory has also been observed 67. In fact, the noncommutative instanton on the torus 68 and the monopole in the noncommutative $U(2)$ Yang-Mills theory 69, 70 have also been studied. It has been demonstrated that the $U(1)$ effective action for branes is the the Dirac-Born-Infeld action [72, and the BPS condition of the ordinary Dirac-Born-Infeld action and a noncommutative action are equivalent in a limit $\alpha^{\prime} \rightarrow 0$ [71. It may be noted that string theory also can give rise to noncommutative gravity [73]. This is done by studding the next to the leading order terms in the Seiberg-Witten limit for the dynamics of closed strings, in the presence of a constant two form field. Thus, the gravitational 
action induced by the bosonic string theory on a space-filling D-brane with a constant magnetic field have been studied in the low energy limit. The induced terms for the interaction vertex of three gravitons on the brane have thus been obtained. It has also been observed that the noncommutative deformations of gravity can lead to a complex metric and in this case the tangent space groups is larger than the Lorentz group 74 . Noncommutativity has also been studied by twisting the diffeomorphism invariance of the general relativity 75 . It may be noted that the deformed algebra corresponding to commutative deformation has been used to construct a covariant tensor calculus for metric, covariant derivatives, curvature and torsion [75. It would be interesting to analyse all these structures using this new form of noncommutativity, which is motivated from gravity's rainbow.

The effect of having an energy dependent metric on the thermodynamics of various black objects has already been studied using gravity's rainbow [16, 19, 17, 33], it would be interesting to analyse such an energy dependence using the approach developed in this paper. Furthermore, the thermodynamics of various interesting black objects has been studied [104, 105, 106, 107, 108, 109, and it would be interesting to analyse the effect of energy dependent noncommutativity on the thermodynamics of such black objects. It is expected that the black hole remnants will not form for all the black objects due to this energy dependent noncommutative deformation of spacetime. It is expected that this deformation will increase the temperature rather than decrease it for all black objects. However, it would be interesting to demonstrate it explicitly for various different black hole solutions. In higher dimensions, interesting solutions to the general relativity exist which have interesting topologies. It is possible for black rings and black saturns to exist in higher dimensions. It will be interesting to analyze the thermodynamics of such solutions using noncommutative formalism. It has also been demonstrated that noncommutativity leads to the existence of a minimum length in spacetime. The black hole thermodynamics for minimum length have been analyzed 110, 111, 112, 113. It will be interesting to analyze a possible link between the thermodynamics of black holes with minimum length and noncommutative black holes. It would also be interesting to generalize this analyse to some energy dependent version of such a deformation. It may be noted that this noncommutative deformation will deform the Heisenberg algebra, and it is known that a deformation of the Heisenberg algebra is related to generalized uncertainty principle, which in turn is related to modified dispersion relation [114, 115. It would be interesting to investigate further the relation between this energy dependent noncommutativity and modified dispersion relation in flat spacetime.

\section{References}

[1] O. J. Rosten, Phys. Rep. 511, 177 (2012)

[2] A. Kaya, Phys. Rev. D 87, 123501 (2013)

[3] K. Groh and F. Saueressig, J. Phys. A 43, 365403 (2010)

[4] J. Magueijo and L. Smolin, Class. Quant. Grav. 21, 1725 (2004)

[5] P. Galan and G. A. M. Marugan, Phys. Rev. D 70, 124003 (2004) 
[6] P. Galan and G. A. M. Marugan, Phys. Rev. D 72, 044019 (2005)

[7] J. Hackett, Class. Quant. Grav. 23, 3833 (2006)

[8] G. 't Hooft, Class. Quant. Grav. 13, 1023 (1996)

[9] V. A. Kostelecky and S. Samuel, Phys. Rev. D 39, 683 (1989)

[10] G. Amelino-Camelia, J. R. Ellis, N. Mavromatos, D. V. Nanopoulos, and S. Sarkar, Nature 393, 763 (1998)

[11] R. Gambini and J. Pullin, Phys. Rev. D 59, 124021 (1999)

[12] S. M. Carroll, J. A. Harvey, V. A. Kostelecky, C. D. Lane and T. Okamoto, Phys. Rev. Lett. 87, 141601 (2001)

[13] M. Faizal, Mod. Phys. Lett. A 27, 1250075 (2012)

[14] M. Faizal, J. Phys. A 44, 402001 (2011)

[15] A. F. Ali and M. M. Khalil, Europhys. Lett. 110, 20009 (2015)

[16] A. F. Ali, Phys. Rev. D 89, 104040 (2014)

[17] A. F. Ali, M. Faizal, and M. M. Khalil, JHEP 1412, 159 (2014)

[18] A. F. Ali, M. Faizal, and M. M. Khalil, Nucl. Phys. B 894, 341 (2015)

[19] A. F. Ali, M. Faizal, M. M. Khalil, Phys. Lett. B743, 295 (2015)

[20] P. Horava, Phys. Rev. D 79, 084008 (2009)

[21] P. Horava, Phys. Rev. Lett. 102, 161301 (2009)

[22] R. Garattini and E. N. Saridakis, Eur. Phys. J. C 75, 343 (2015)

[23] F. Girelli, S. Liberati and L. Sindoni, Phys. Rev. D 75, 064015 (2007)

[24] G. G. Carvalho, I. P. Lobo and E. Bittencourt, Phys. Rev. D 93, 044005 (2016)

[25] I. P. Lobo, N. Loret and F. Nettel, Phys. Rev. D95, 046015 (2017)

[26] I. P. Lobo, N. Loret and F. Nettel, Eur. Phys. J. C77, 451 (2017)

[27] L. Barcaroli, L. K. Brunkhorst and G. Gubitosi, N. Loret and C. Pfeifer, Phys. Rev. D 96, 084010 (2017)

[28] Y. Gim and W. Kim, JCAP 1410, 003 (2014)

[29] Y. Gim and W. Kim, Eur. Phys. J. C 76, 166 (2016)

[30] S. H. Hendi, Gen. Relativ. Gravit. 48, 50 (2016)

[31] P. Rudra, M. Faizal, A. F. Ali, Nucl. Phys. B 909, 725 (2016)

[32] S. H. Hendi, S. Panahiyan, B. E. Panah, M. Faizal and M. Momennia, Phys. Rev. D 94, 024028 (2016) 
[33] S. H. Hendi and M. Faizal, Phys. Rev. D 92, 044027 (2015)

[34] Y. Ling, X. Li, and H. b. Zhang, Mod. Phys. Lett. A 22, 2749 (2007)

[35] H. Li, Y. Ling, and X. Han, Class. Quant. Grav. 26, 065004 (2009)

[36] J. D. Bekenstein, Phys. Rev. D 7 , 2333 (1973)

[37] J. D. Bekenstein, Phys. Rev. D 9, 3292 (1974)

[38] S. W. Hawking, Nature 248, 30 (1974)

[39] S. W. Hawking, Commun. Math. Phys. 43, 199 (1975)

[40] N. Altamirano, D. Kubiznak, R. B. Mann, and Z. Sherkatghanad, Galaxies $2,89(2014)$

[41] L. Susskind, J. Math. Phys. 36, 6377 (1995)

[42] R. Bousso, Rev. Mod. Phys. 74, 825 (2002)

[43] D. Bak and S. J. Rey, Class. Quant. Grav. 17, L1 (2000)

[44] S. K. Rama, Phys. Lett. B 457, 268 (1999)

[45] A. Ashtekar, Lectures on Non-perturbative Canonical Gravity, World Scientific (1991)

[46] T. R. Govindarajan, R. K. Kaul, V. Suneeta, Class. Quant. Grav. 18, 2877 (2001)

[47] S. Carlip, Class. Quant. Grav. 17, 4175 (2000)

[48] T. R. Govindarajan, R. K. Kaul and V. Suneeta, Class. Quant. Grav. 18, $2877(2001)$

[49] S. N. Solodukhin, Phys. Rev. D57, 2410 (1998)

[50] A. Sen, JHEP, 04, 156 (2013)

[51] A. Sen, Entropy 13, 1305 (2011)

[52] D. A. Lowe and S. Roy, Phys. Rev. D82, 063508 (2010)

[53] J. Jing and M. L Yan, Phys. Rev. D63, 24003 (2001)

[54] K. Nozari and S. Islamzadeh, Astrophys. Space Sci. 347, 299 (2013)

[55] S. H. Mehdipour and A. Keshavarz, Europhys. Lett. 98, 10002 (2012)

[56] M. Sharif and Wajiha Javed, Can. J. Phys. 89, 1027 (2011)

[57] P. Nicolini and G. Torrieri, JHEP 1108, 097 (2011)

[58] S. Minwalla, M. Van Raamsdonk and N. Seiberg, JHEP 0002, 020 (2000)

[59] J. W. Moffat, Phys. Lett. B491, 345 (2000)

[60] J. W. Moffat, Phys. Lett. B493, 142 (2000) 
[61] E. Witten, Nucl. Phys. B460 , 335 (1996)

[62] A. Connes, M.R. Douglas and A. Schwarz, JHEP 02, 003 (1998)

[63] T. Kawano and K. Okuyama, Phys. Lett. B433, 29 (1998)

[64] M.R. Douglas and C. Hull, JHEP 02, 008 (1998)

[65] Y.E. Cheung and M. Krogh, Nucl. Phys. B528, 185 (1998)

[66] N. Nekrasov and A. Schwarz, Commun. Math. Phys. 198, 689 (1998)

[67] M. Berkooz, Phys. Lett.B430, 237 (1998)

[68] A. Astashkevich, N. Nekrasov and A. Schwarz, Commun. Math. Phys. 211, $167(2000)$

[69] K. Hashimoto, H. Hata and S. Moriyama, JHEP 9912, 021 (1999)

[70] A. Hashimoto and K. Hashimoto, JHEP 11, 005 (1999)

[71] N. Seiberg and E. Witten, JHEP 09, 032 (1999)

[72] A. A. Tseytlin, The many faces of the superworld, World Scientific 417 (1999)

[73] L. Alvarez-Gaume, F. Meyer and M. A. Vazquez-Mozo, Nucl. Phys. B753, $92(2006)$

[74] A. H. Chamseddine, Phys. Rev. D 69, 024015 (2004)

[75] P. Aschieri, C. Blohmann, M. Dimitrijevic, F. Meyer, P. Schupp and J. Wess, Class. Quant. Grav. 22, 3511 (2005)

[76] J. E. Moyal, Proc. Camb. Phil. Soc. 45, 99 (1949)

[77] J. Madore, S. Schraml, P. Schupp and J. Wess, Eur. Phys. J. C16, 161 (2000)

[78] H. Weyl, The Theory of Groups and Quantum Mechanics, Dover, New-York (1931)

[79] S. C. Ulhoa, R. G. G. Amorim and A. F. Santos, Gen. Rel. Grav. 46, 1760 (2014)

[80] M. Kober, Int. J. Mod. Phys. A 30, 1550085 (2015)

[81] J. W. Moffat, Phys. Lett. B 506, 193 (2001)

[82] E. C. Young, D. Lee and Y. Lee, Class. Quant. Grav. 26, 185001 (2009)

[83] Omer F. Dayi and A. Jellal, J. Math. Phys. 51, 063522 (2010)

[84] M. Schnabl, JHEP 0011, 031 (2000)

[85] O. Andreev and H. Dorn, Phys. Lett. B 476, 402 (2000)

[86] C. F. Farias, A. C. Lehum, J. R. Nascimento and A. Y. Petrov, Phys. Rev. D 86, 065035 (2012) 
[87] A. C. Lehum, J. R. Nascimento, A. Y. Petrov and A. J. d. Silva, Phys. Rev. D 88, 045022 (2013)

[88] D. Bak and K. Lee, Phys. Lett. B 495, 231 (2000)

[89] B. Charneski, A. F. Ferrari and M. Gomes, J. Phys. A 40, 3633 (2007)

[90] E. Akofor, A. P. Balachandran, S. G. Jo and A. Joseph, JHEP 0708, 045 (2007)

[91] G. Ahuatzin, I. Bautista, J. A. Hernandez-Lopez, F. Ramirez-Zavaleta and J. J. Toscano, Phys. Rev. D 82, 053001 (2010)

[92] G. Amelino-Camelia, J. R. Ellis, N. E. Mavromatos and D. V. Nanopoulos, Int. J. Mod. Phys. A 12, 607 (1997)

[93] J. Magueijo and L. Smolin, Phys. Rev. Lett. 88, 190403 (2002)

[94] E. Greenwood and D. Stojkovic, JHEP 0909, 058 (2009)

[95] R. Parentani, Phys. Rev. D 63, 041503 (2001)

[96] R. Schutzhold and W. G. Unruh, Phys. Rev. D 81, 124033 (2010)

[97] A. Almheiri, D. Marolf, J. Polchinski and J. Sully, JHEP 1302, 062 (2013)

[98] L. Susskind, L. Thorlacius and J. Uglum, Phys. Rev. D 48, 3743 (1993)

[99] L. Susskind and L. Thorlacius, Phys. Rev. D 49, 966 (1994)

[100] C. R. Stephens, G. 't Hooft and B. F. Whiting, Class. Quant. Grav. 11, $621(1994)$

[101] Y. Gim and W. Kim, JCAP 05, 002 (2015)

[102] A. F. Ali, M. Faizal, B. Majumder and R. Mistry, Int. J. Geom. Meth. Mod. Phys. 12, 1550085 (2015)

[103] A. F. Ali, M. Faizal and B. Majumder, Europhys. Lett. 109, 20001 (2015)

[104] N. Altamirano, D. Kubiznak, R. B. Mann and Z. Sherkatghanad, Galaxies 2,89 (2014)

[105] M. M. Caldarelli, R. Emparan and M. J. Rodriguez, JHEP 0811, 011 (2008)

[106] J. P. S. Lemos, F. J. Lopes, M. Minamitsuji and J. V. Rocha, Phys Rev D 92, $064012(2015)$

[107] P. Figueras and S. Tunyasuvunakool, JHEP 1503, 149 (2015)

[108] J. S. Schiffrin and R. M. Wald, Class. Quant. Grav. 31, 035024 (2014)

[109] C. Herdeiro, E. Radu and C. Rebelo, Phys. Rev. D 81, 104031 (2010)

[110] S. Gangopadhyay, A. Dutta and A. Saha, Gen. Rel.Grav. 46, 1661 (2014)

[111] L. Xiang and X. Q. Wen, JHEP 0910, 046 (2009) 
[112] M. Faizal and M. Khalil, Int. J. Mod. Phys. A 30, 1550144 (2015)

[113] S. Gangopadhyay, A. Dutta and M. Faizal, Europhys. Lett. 112, 20006 (2015)

[114] G. Amelino-Camelia, M. Arzano, Y. Ling and G. Mandanici, Class. Quant. Grav. 23, 2585 (2006)

[115] K. Nozari and B. Fazlpour, Gen. Rel. Grav. 38, 1661 (2006) 